\title{
Roles of Fibroblast Growth Factors and Their Therapeutic Potential in Treatment of Ischemic Stroke
}

\author{
Confidence Dordoe ${ }^{1 \dagger}$, Keyang Chen ${ }^{1,2 \dagger}$, Wenting Huang ${ }^{3}$, Jun Chen ${ }^{1}$, Jian $\mathrm{Hu}^{1}$, Xue Wang ${ }^{1 *}$ \\ and $\operatorname{Li~Lin}^{1,4 *}$ \\ ${ }^{1}$ School of Pharmaceutical Sciences, Wenzhou Medical University, Wenzhou, China, ${ }^{2}$ Department of Neurology, The Second \\ Affiliated Hospital and Yuying Children' Hospital of Wenzhou Medical University, Wenzhou, China, ${ }^{3}$ School of the First Clinical \\ Medical Sciences, Wenzhou Medical University, Wenzhou, China, ${ }^{4}$ Research Units of Clinical Translation of Cell Growth Factors \\ and Diseases Research, Chinese Academy of Medical Science, Beijing, China
}

OPEN ACCESS

Edited by:

Yidong Wang,

Xian Jiaotong University, China

Reviewed by:

Ning Liu,

Tulane University, United States

Feilin Liu,

Mayo Clinic Florida, United States

Changhong Xing,

University of Texas Southwestern

Medical Center, United States

*Correspondence:

Xue Wang

xinyuw001@163.com

Li Lin

linliwz@163.com

${ }^{t}$ These authors have contributed equally to this work

Specialty section:

This article was submitted to

Neuropharmacology,

a section of the journal

Frontiers in Pharmacology

Received: 24 February 2021

Accepted: 31 March 2021

Published: 22 April 2021

Citation:

Dordoe C, Chen K, Huang W, Chen J, Hu J, Wang X and Lin L (2021) Roles of Fibroblast Growth Factors and Their Therapeutic Potential in Treatment of Ischemic Stroke.

Front. Pharmacol. 12:671131. doi: 10.3389/fphar.2021.671131
Stroke is the leading cause of death worldwide, and its treatment remains a challenge. Complex pathological processes are involved in stroke, which causes a reduction in the supply of oxygen and energy to the brain that triggers subsequent cascade events, such as oxidative stress, inflammatory responses and apoptosis, resulting in brain injury. Stroke is a devastating disease for which there are few treatments, but physical rehabilitation can help improve stroke recovery. Although there are very few treatments for stroke patients, the discovery of fibroblast growth factors (FGFs) in mammals has led to the finding that FGFs can effectively treat stroke in animal models. As presented in this review, FGFs play essential roles by functioning as homeostatic factors and controlling cells and hormones involved in metabolism. They could be used as effective therapeutic agents for stroke. In this review, we will discuss the pharmacological actions of FGFs on multiple targets, including their ability to directly promote neuron survival, enhance angiogenesis, protect against blood-brain barrier (BBB) disruption, and regulate microglial modulation, in the treatment of ischemic stroke and their theoretical mechanisms and actions, as well as the therapeutic potential and limitations of FGFs for the clinical treatment of stroke.

Keywords: fibroblast growth factors, stroke, therapeutic potential, theoretical mechanism, clinical application

\section{INTRODUCTION}

Stroke is defined as an acute cerebrovascular disease attributed to sudden rupture of blood vessels in the brain or blockage of blood vessels that prevent blood from flowing to the brain (Campbell and Khatri, 2020). The prevalence of stroke is on the rise, and it has been reported to be one of the most common causes of mortality and morbidity worldwide (Causes of Death Collaborators, 2017), and the number of individuals living with its effects are high due to growing and aging of the population (Stinear et al., 2020). For a long time, intravenous recombinant tissue plasminogen activator (rt-PA, alteplase) has been the exclusive therapeutic drug for acute ischemic stroke. Recent advances in mechanical clot retrieval strategies, such as mechanical thrombectomy for the treatment of large artery stroke, allow effective recanalization and have resulted in improvements in patient outcomes (Prabhakaran et al., 2015; Griessenauer et al., 2018). However, a narrow therapeutic window limits the benefits of these strategies. Moreover, the main strategy for secondary stroke prevention is the use of different pharmacological agents, mainly antiplatelets and anticoagulants, but half of patients have an increased risk of recurrent stroke (Arsava et al., 2016). Stroke has been reported to cause 
inflammation, and activation of the innate immune system is involved in its pathogenesis (Zuo et al., 2020). Neurorestorative progression in stroke is characterized by neurogenesis, angiogenesis, and synaptic plasticity, which are beneficial for functional recovery (Chen et al., 2019). Newly generated blood vessels increase cerebral flow in the ischemic boundary to supply oxygen and nutrients to the ischemic area to improve neurological function (Beck and Plate, 2009). Therefore, the enhancement of angiogenesis may become a promising therapeutic strategy for ischemic stroke treatment.

The blood-brain barrier (BBB) is composed of endothelial cells (ECs), pericytes, astrocyte end-feet, and a basement membrane. Brain ECs that are connected by tight junction proteins and adhesive proteins (e.g., Occludin, $\mathrm{ZO}-1$, and Claudin-5) protect $\mathrm{BBB}$ integrity. Following ischemic stroke, the $\mathrm{BBB}$ is acutely disrupted, resulting in secondary brain injury due to cerebral edema and the infiltration of peripheral immune cells into the central nervous system (CNS). Accumulating evidence indicates that targeting the $\mathrm{BBB}$ may be a promising therapeutic strategy for the treatment of ischemic stroke (Jiang et al., 2018). On the other hand, the production of damage-associated molecular patterns (DAMPs) by neurons and glial cells are dramatically increased after ischemic stroke. Ischemic stroke induces the activation of astrocytes and microglia, the production of proinflammatory cytokines and chemokines and further exacerbation of tissue damage. Therefore, suppression of proinflammatory cytokine expression or promotion of antiinflammatory cytokine expression by microglial regulation may be another promising treatment for ischemic stroke (Wang et al., 2020).

Stroke is a neurological disease with poor prognosis. The ultimate goal of stroke treatment is to promote neurological function. The subventricular zone (SVZ) and subgranular zone are known to contribute to functional recovery after stroke through the process of neurogenesis (Rahman et al., 2021). Accumulating evidence suggests that promoting neurogenesis in the chronic phase of ischemic stroke retards disease progression and improves neurological dysfunction (Freret et al., 2006; Bonsack et al., 2020). Therefore, enhancing neurogenesis has become an attractive approach promoting recovery of in the chronic phase of stroke.

There are serious barriers to the clinical translation of drug treatments for stroke. More than 1,000 therapeutic drugs that were shown to have preclinical therapeutic potential for the treatment ischemic brain injury failed in clinical trials (Candelario-Jalil and Paul, 2021). There are still no effective therapeutic strategies that have been shown to improve outcomes after stroke. The complexity of stroke and its associated comorbidities may limit the effectiveness of neuroprotective drugs.

Fibroblast growth factors (FGFs) are polypeptide growth factors involved in numerous processes, such as growth, development, neuronal functions, metabolism, proliferation, migration, apoptosis, wound repair, and angiogenesis (Itoh and Ornitz, 2011). In humans, FGFs support blood vessels to help supply of nutrients to the brain and other organs (Matkar et al., 2017). Similarly, their homeostatic functions enable them to repair tissues, accelerate wound healing and control the nervous system (Beenken and Mohammadi, 2009). The angiogenic and neurotrophic characteristics of FGFs suggest that they may be effective therapeutic agents for ischemic stroke treatment. Many studies have shown that some FGFs are associated with stroke. In this review, we summarize the potential roles of FGFs in promoting neural protection, neuroregeneration, vascular protection, angiogenesis, and $\mathrm{BBB}$ protection after ischemic stroke and suggest that FGFs may be candidate agents for improving stroke outcome through multiple pathways.

\section{FGF FAMILY}

The FGF family is made up of signaling and nonsignaling proteins that are structurally related and grouped into 6 subfamilies based on their properties and sequences (Itoh and Ornitz, 2011; Ornitz and Itoh, 2015). The FGF1 subfamily includes FGF1 and FGF2; the FGF4 subfamily comprises FGF4, FGF5, and FGF6; the FGF7 subfamily includes FGF3, FGF7, FGF10, and FGF22; the FGF8 subfamily comprises FGF8, FGF17, and FGF18; the FGF9 subfamily includes FGF9, FGF16, and FGF20; the FGF11 homologous subfamily comprises FGF11, FGF12, FGF13, and FGF14; and the FGF15/19 subfamily includes FGF15/19, FGF21, and FGF23 (Krejci et al., 2009). The FGF family exerts survival-promoting and protective effects to promote neural outgrowth and neurogenesis in the brain (Mudo et al., 2009). Signaling FGFs are expressed in nearly all tissues, and they play roles in embryonic development and organogenesis at the onset of development and function as homeostasis factors for repair, regeneration and maintenance in adults (Ornitz and Itoh, 2001). Nonsignaling FGFs are called intracellular FGFs because they serve as cofactors for the regulation of voltage-gated sodium channels and other molecules, making them essential regulators of neuronal and myocardial excitability (Goldfarb, 2005). The various characteristics of FGF family members and their receptors make them attractive targets for drug development (Belov and Mohammadi, 2013). Members of five other subfamilies, i.e., the FGF1, FGF4, FGF7, FGF8, and FGF9 subfamilies, have paracrine functions; however, FGF9, FGF16, and FGF20 have bipartite signal sequences that are not cleaved (Revest et al., 2000). The table below provides a summary of the FGF subfamilies, their expression sites and their functions (Table 1).

\section{FIBROBLAST GROWTH FACTORS IN THE TREATMENT OF STROKE \\ FGF1 in Stroke}

The administration of FGF1 is by a non-invasive method, where it is intranasally delivered into the CNS because it cannot pass through the BBB (Cheng et al., 2011). This intranasal delivery enhanced peripheral nerve regeneration in vivo (Jacques et al., 1999) with protective effect against neurofunctional deficit shown to be partially regulated by PI3K/Akt (Wu et al., 2017). Furthermore, FGF1 administered in middle cerebral artery 
TABLE 1 | FGF subfamily and their function.

FGF

subfamily

FGF 1 subfamily

FGF $1 \quad$ Brain, pituitary, nerve tissue, retina, adrenal gland, heart, and bone

FGF $2 \quad$ Various tissues and organs derived from mesoderm and neuroectoderm, and tumor tissues

FGF 4 subfamily

FGF $4 \quad$ Posterior part of the limb buds

FGF $5 \quad$ Brain

FGF $6 \quad$ Developing skeletal muscle

FGF 7 subfamily

FGF $3 \quad$ Mammary tumors

FGF $7 \quad$ Fetal lung mesenchymal tissue

FGF $10 \quad$ First observed in the limb bud

FGF $22 \quad$ Mammalian brain and skin wounds

FGF 8 subfamily

FGF 8 Progenitor cells in the midbrain and hindbrain

FGF $17 \quad$ Cortex

FGF $18 \quad$ Skin and cortical neurons

FGF 9 subfamily

FGF $9 \quad$ Neurons in the cortex hippocampus, thalamus, cerebellum, spinal cord, epithelium and mesothelium

FGF 16 Embryonic brown adipose tissue, and the inner ear

FGF $20 \quad$ Brain

FGF 15/19 subfamily

FGF 15 Absorptive cells of the mouse ileum

FGF 19 Absorptive cells of the human ileum; can be found in the brain, skin, retina, gallbladder, small intestine, kidney and umbilical cord

FGF $21 \quad$ Muscle, liver, islet $\beta$-cells in the pancreas and thymus adipose tissue

FGF 23 Bone, lung, brain, heart, muscle and spleen

FGF homologous family

FGF $11 \quad$ Neuroblastoma, retinoblastoma and brain tumors

FGF $12 \quad$ Brain, eye, heart and testis

FGF $13 \quad$ Brain and heart

FGF 14
Adult cerebellum

Function

References

Promotes mitosis, wound healing, angiogenesis, hematopoiesis, tumorigenesis, and neurogenesis Promotes mitosis vascular remodeling, bone formation, pulmonary fibrosis, neurodevelopment, and tumor metabolism

HST-1; is involved in limb development and internal organs development

Is involved in hair follicle development, regulates neuronal differentiation and survival and regulates GFAP expression HST-2; is involved in myogenesis and muscle regeneration

Controls the inner ear plan

KGF; prevents lung branch formation, and lung inflammation Knockout mice, show absence of lungs and complete resection of the fore and hind limbs, promotes the proliferation of mammary gland epithelial cells and reduced apoptosis

Presynaptic molecule; is involved in repair, stimulates the formation of inhibitory presynaptic terminal, alleviates depression, and is involved in vesicle clustering, and skin development

AIGF; sets up and maintains the midbrain border and regulates the growth and differentiation of progenitor cells to generate midbrain and hindbrain structures

Has similarities with FGF8; acts as an autocrine growth factor in neoplastic prostate epithelial cells and is involved in neocortex development

Promotes chondrogenesis, cortical neurons, skin repair, and neuroprotection

Stimulates glial cell growth, is involved in fetal lung development, and enhances the survival of acetylcholinesterase (AChE)-positive neurons Is involved in proliferation of embryonic brown adipose tissue and fate determination of otic cells

Enhances the survival of midbrain dopaminergic neurons and protects against PD.

Is involved in feedback inhibition of hepatic bile acid synthesis and regulates glucose and lipid metabolism

Acts as a hormone to protect against infarction in response to bile acid absorption, regulates glucose and lipid metabolism, and nonmitogenic effects

Plays important role in glucose and lipid metabolism and protects the cardiovasculature in the heart

Regulates phosphate concentration in plasma, decreases absorption and increases the excretion of phosphate

Expression is in ECs by HIF-1a; stimulates capillary-like endothelial tube formation, which is associated with angiogenesis

Contributes to skeletal growth and developmental failure in grade II and III kashin-beck disease (KBD).

Is involved in neural differentiation in xenopus early development and controls. proliferation and differentiation of skeletal muscle

Regulates intrinsic excitability of cerebellum purkinje neurons
Cheng et al. (2011), Zou et al., (2020)

Charoenlarp et al. (2017), Koo et al. (2018)

Robertson et al. (1997), Okunieff et al. (2003)

Lindholm et al. (1994), Reuss

et al. (2003)

de Lapeyriere et al. (1990)

Dickson et al., (1989)

Finch and Rubin (2004)

Cui and Li (2013)

Beyer et al. (2003), Xu et al. (2017)

Lim et al. (2015)

Polnaszek et al. (2004)

Ellsworth et al. (2004)

Kanda et al. (2000), Ellsworth et al. (2004), Lum et al. (2009)

Konishi et al. (2000)

Boshoff et al. (2018), Xu et al. (2018)

Potthoff et al. (2011), Huang et al. (2018)

Nishimura et al. (1999), Potthoff et al. (2011)

Chen et al. (2018), Wang et al. (2018)

Kendrick et al. (2011), Ix et al.

(2012), Zheng et al. (2020)

Lee et al. (2017)

Zhang et al. (2016)

Lu et al. (2015), Yue et al. (2018)

Shakkottai et al. (2009) 


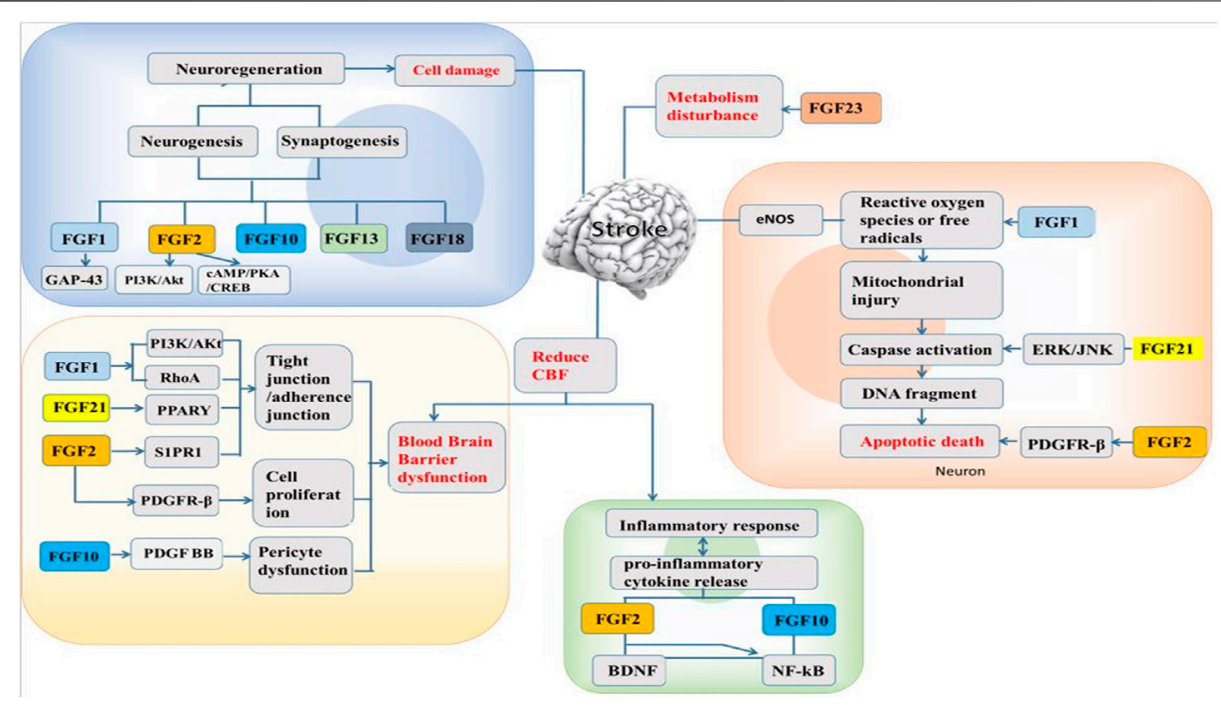

FIGURE 1 | Pharmacological effect of FGFs on stroke and its possible mechanism.

occlusion (MCAO) after 14 days of ischemia insult was seen that, the cerebral infarction area was reduced and the number of blood vessels increased as well (Xu et al., 2009). Intranasal nonmitogenic FGF1 (nm-FGF1) administration for 10 consecutive days enhanced angiogenesis via sphingosine-1-phosphate receptor 1 (S1P1) signaling pathway following stroke (Zou et al., 2020). The mixture of FGF1 and fibrin glue (a slowrelease carrier and adhesive agent) in the CNS when applied topically after injury reduced the cerebral infarction, protects cortical cells from loss and reduce microglia penetration thereby promoting a recovery system (Tsai et al., 2015). Treatment with FGF1 preceded in ischemia preserved $\mathrm{BBB}$ integrity by regulating tight junctions and adherens junctions' expressions (Cheng et al., 2011; Chen et al., 2020). In addition, brain cells with FGF1 exhibited protective effects by upregulating tight junction proteins and inhibiting RhoA through PI3K-Akt-Rac1 pathway regulation to protect the BBB (Wu et al., 2017). From the above, application of FGF1 after stroke create a favorable condition to regulate the injury from neuronal regeneration, neuroprotection, angiogenesis, and BBB protection (Figure 1).

\section{FGF2 in Stroke}

FGF2, also called basic fibroblast growth factor (bFGF), was first purified from the bovine pituitary gland in 1975 (Gospodarowicz, 1975) and is widely expressed in the CNS, especially in the hippocampus (Woodbury and Ikezu, 2014). FGF2 is mainly expressed in neurons and glial cells during puberty and adulthood. It is a polypeptide that has potent trophic and protective effects on the brain and confers neuroprotection after brain injury (Wada et al., 2003). When the expression of FGF2 in the nervous system is high, it shows that FGF2 has neuroprotective effects in animal models of stroke (Kawamata et al., 1997). Reports have proven that FGF2 exerts a neuroprotective effect after stroke (Zhao et al., 2016). FGF2 enhances functional recovery after stroke by promoting progenitor cell proliferation, migration and differentiation in the brain (Bao et al., 2011). Transfection of mesenchymal stromal cells (MSCs) with a herpes simplex virus type 1 (HSV-1) vector expressing FGF2 promotes functional recovery and leads to a reduction in the infarct volume in rats after MCAO (Ikeda et al., 2005). Several other reports have demonstrated a significant reduction in infarct volume in the FGF2-treated group compared to the group that received vehicle treatment only (Ay et al., 1999; Li and Stephenson, 2002). Interestingly, animals given FGF2 show significant improvement, supporting the claim that FGF2 enhances neurogenesis, which contributes to recovery after ischemia (Sun et al., 2009). Platelet-derived growth factor receptor $\beta$ (PDGFR $\beta$ ), which is expressed in pericytes and pericyte-derived fibroblast-like cells, plays important roles in the maintenance of the BBB (Hutter-Schmid and Humpel, 2016). It has been reported that the expression of PDGFR $\beta$ is markedly increased in pericytes after ischemic insult (Shibahara et al., 2020). FGF2 increases the number of pericytes to promote pericyte functions via its interaction with PDGF-BB, thus exerting neuroprotective and angiogenic effects (Nakamura et al., 2016). Moreover, injury induces the expression of FGF2 in reactive glial cells to promote synthesis and enhance cell proliferation and neuronal survival; thus, FGF2 has a neuroprotective effect in the brain and maintains the BBB (Wang Z. G. et al., 2016). Sphingosine-1-phosphate (S1P) is a member of sphingolipid family known for regulating cellular processes such as cell growth, angiogenesis and survival (Iwasawa et al., 2018). Following oxygen-glucose deprivation/reperfusion (OGD/R) in human microvascular endothelial cell monolayers, exogenous administration of FGF2 prevents BBB damage by upregulating sphingosine-1-phosphate receptor-1 (S1PR1) protein expression (Lin et al., 2018). Recent studies reported that when the S1P1 is activated, it could ameliorate injuries in MCAO models, and also the S1PR1 modulators involved in S1P1 signaling pathway could restore microvascular circulation to the cerebral 
ischemia (Li et al., 2019; Zou et al., 2020). In Lin et al. report, they demonstrated the protective ability of recombinant FGF2 on $\mathrm{BBB}$ integrity in OGD/R-induced endothelial monolayer permeability, and it was shown that the protective effect of FGF2 was mediated via S1PR1 upregulation (Lin et al., 2018). These results indicate that FGF2 can improve functional recovery and reduce the infarct volume after stroke through various mechanisms. Zhao et al. has demonstrated that FGF2 possess neuroprotective effects through PI3K/Akt activation in a rodent stroke model Zhao et al. (2016). A recent study on the mechanism underlying the effects of cyclic adenosine monophosphate (cAMP)/protein kinase A (PKA) on ischemic injury depends on regulating apoptosis and inflammation, and FGF2-mediated cAMP/PKA/cAMPresponse element binding protein (CREB) pathway to promote dendritic and synaptic plasticity (Li et al., 2020). Besides, It has been reported that FGF2 decreased the levels of pro-inflammatory cytokines (IL- 6 and TNF- $\alpha$ ), through the regulation of the upstream toll-like receptor 4/nuclear factor $\kappa \mathrm{B}$ (TLR4/NFkB) signaling pathway (Ye et al., 2015) (Figure 1).

\section{FGF21 in Stroke}

FGF21 is expressed in different tissues and organs, such as the liver, pancreas, skeletal muscle, thyroid and adipose tissue (Nishimura et al., 2000; Watanabe et al., 2020). FGF21 exerts its activity by binding to FGFR and $\beta$-klotho (KLB). It has the ability to regulate endocrine processes during glucose and lipid metabolism to aid the production and consumption of energy (Inagaki et al., 2007; Chau et al., 2010). Majority of stroke patients have type 2 diabetes (T2D). Recent studies showed that T2D affects the integrity of $\mathrm{BBB}$ and implicates the $\mathrm{BBB}$ permeability state after ischemic stroke, but the use of recombinant FGF21 (rFGF21) has been beneficial to the treatment of stroke mice with T2D (Jiang et al., 2020). A report has shown that FGF21 is a suitable mediator for adaptive responses to tissue injury, suggesting it to be a novel therapeutic agent that has a protective ability against stroke stresses in T2D that could improve the neurological outcomes (Jiang et al., 2018). Also, delayed recanalization is another promising alternative for stroke patients who could not meet the window time after stroke injury (Kelly and Holloway, 2018). The delay increases endogenous FGF21 in the penumbra when administered, thereby decreasing the neuronal apoptosis for the enhancement of a better neurological outcome through FGF21/FGFR1/PI3K/caspase-3 signaling pathway (Zheng et al., 2019). Some reports showed that FGF21 plays a significant role in cardiocerebrovascular disease and has the ability to prevent arteriosclerosis by suppressing the hepatic sterol regulatory element (Lin et al., 2015; Wu et al., 2020). A recent report revealed that FGF21 has a low binding affinity for heparan and therefore has a tendency to pass through the BBB (Chen et al., 2018). The peroxisome proliferator-activator receptor gamma $(\operatorname{PPAR} \gamma)$ signaling pathway is one of the important downstream pathways of FGF21 and regulates the transcription of genes known to be involved in adipocyte growth and differentiation (Dutchak et al., 2012). Studies have shown that primary neurons expressing FGF21 have a neuroprotective effect against excitotoxicity induced by glutamate (Leng et al., 2015). Lyophilized recombinant human FGF21 (rhFGF21) protects against cerebral ischemia in MCAO rats and neuronal cells by decreasing endoplasmic reticulum (ER) stress (Yang et al., 2018). Ischemia/reperfusion (I/R) was shown to inhibit the activity of endogenous antioxidant enzymes and promote the overproduction of ROS (Lo et al., 2003), ultimately leading to cellular apoptosis (Fleury et al., 2002). Wan et al. found that FGF21 is involved in the signaling pathway responsible for I/R-mediated hippocampal injury (Wan et al., 2019). FGF21 protects against hypoxia stress-induced injury in cerebral microvascular ECs by inducing heat shock protein expression (Wang et al., 2019). Wang et al. found that FGF21 protects against Ang II-induced cerebrovascular aging in ischemia by improving mitochondrial biogenesis and inhibiting p53 activation in an AMPK-dependent manner (Wang X. M. et al., 2016). FGF21 was shown to alleviate MCAO-induced brain injury via activation of PI3K/Akt and inhibition of GSK$3 \beta$ (Wang et al., 2016). Furthermore, FGF21 decreases the expression of ER stress-related proteins in MCAO rats and PC12 cells (Yang et al., 2018). In addition, in our recent study, we discovered that rhFGF21 treatment alleviates motor nerve dysfunction by modulating microglia/macrophage-mediated neuroinflammation via inhibition of NF- $\kappa \mathrm{B}$ signaling pathways (Wang et al., 2020). Overall, FGF21 protects against stroke through actions affecting multiple targets, including promotion of neuronal survival and induction of ER stress and microglia/macrophage-mediated neuroinflammation reductions (Figure 1).

\section{Other FGFs in Stroke}

In addition, other FGFs exhibit potential pharmacological actions against stroke. FGF10 belongs to the FGF7 subfamily and was first cloned in rat embryos (Yamasaki et al., 1996). In vitro findings have concluded that exogenous FGF10 is expressed in neurons but not in astrocytes because it is found at levels content in neuronal culture medium and exerts protective effects in neurons deprived of oxygen and glucose (Li et al., 2015). In another study, it was reported that neuron-derived FGF10 can inhibit NF- $\kappa$ Bdependent neuroinflammation and promote neuronal survival by activating the PI3K/Akt signaling pathway in an MCAO mouse model (Li et al., 2016). Comparative genome analysis of strokerelated gene expression profiles has revealed that combination treatments may cause overexpression of the FGF12 gene (Liu et al., 2012). FGF13, which belongs to a homologous FGF subfamily and has a molecular weight of $22 \mathrm{kDa}$, is distributed widely throughout the developing brain (Lu et al., 2015). Studies have proven that intravenous administration of FGF13 is capable of reducing the infarct volume and brain swelling and alleviating focal cerebral ischemia. The possible mechanism of action of FGF13 may be similar to that of FGF2 (Yao et al., 1999). FGF18 is mainly expressed in the brains of developing embryos (Maruoka et al., 1998). Reports have shown that FGF18 stimulates neurite outgrowth (Ohbayashi et al., 1998) and has mitogenic effects on glial cells and astrocytes (Hoshikawa et al., 2002). Ellsworth and collaborators assessed the neuroprotective effects of FGF18 in MCAO rats and the appropriate treatment time window 
(Ellsworth et al., 2003; Ellsworth et al., 2004). The results revealed that FGF18 induced a reduction in the infarct volume and improvements in motor ability and exploratory behavior associated with increased cerebral blood flow. FGF18 might be more efficacious than FGF2. FGF18 administration may be effective at both early and later time points. FGF23 is a bonederived hormone that is expressed in tissues such the lungs, heart, brain, muscles, and spleen and helps ameliorate hyperphosphatemia in patients (Taylor et al., 2011; van Venrooij et al., 2014). A high level of FGF23 in the blood results in a greater risk of cardiovascular disease and stroke (Wright et al., 2014). Previous studies have reported that higher FGF23 expression is associated with a higher risk of stroke (Panwar et al., 2015; Jiang et al., 2016). A health study report revealed that FGF23 is independently associated with a higher risk of stroke (Ix et al., 2012). However, Kendrick's research and the Northern Manhattan Study (NOMAS) reported opposite findings (Kendrick et al., 2011; Wright et al., 2014). This discrepancy might have resulted from the differences in the associations between FGF23 and stroke subtypes, such as ischemic stroke and hemorrhagic stroke (Yao et al., 2018). Furthermore, higher FGF23 expression exacerbates atherosclerosis (Coban et al., 2018) and toxicity to vessels, leading to the pathophysiology of stroke (Fandler-Höfler et al., 2019). In addition, only one study has focused on FGF7, which exerts a protective effect against ischemic hippocampal neuron damage (Sadohara et al., 2001).

\section{PROGRESS IN FGF RESEARCH FOR CLINICAL STROKE TREATMENT}

\section{Therapeutic Potential}

Although the therapeutic potential of FGFs in stroke has been indicated by studies in animal models, it has not been reported in the clinic. The main factors that impede the use of FGF in trials is the difficulty in the translation of doses and targeting of the BBB. A phase II/III safety and efficacy trial of FGF2 showed that FGF2 can likely be given safely to stroke patients. The ideal time window for the administration of this agent may exceed $5 \mathrm{~h}$ after stroke (Bogousslavsky et al., 2002). However, another study performed in North America was terminated by the sponsor at the advice of an independent data and safety monitoring committee because the incidence of adverse neurological outcomes and mortality was higher in the active treatment groups than the control group (Clark et al., 2000). The most common adverse events associated with FGF2 treatment during and within 2 days after the infusion period were fever, leukocyte activation, vomiting and hypokalemia. Similarly, the intravenous delivery of FGF2 or placebo in the European-Australian phase II/ III trial on the 286 acute ischemic stroke patients for more than $24 \mathrm{~h}$ showed no significant neuroprotection, but rather caused hypotension and high death rate in treated patients (Bogousslavsky et al., 2002). The basis for the clinical trials of FGF2 were as a result of the improvements shown in the animal models such as the reduction of infarct size, cell proliferation, apoptosis, and improved survival of new mature neurons
(Lanfranconi et al., 2011). FGF2 clinical failure raised concerns about the significance of $\mathrm{BBB}$ in achieving a remarkable therapeutic level in the brain, and reducing the adverse effects in peripheral tissues. One possible way to consider delivering therapeutic agents to the brain to avoid peripheral side effects is by intranasal administration; thus, a non-invasive method to bypass the BBB into the CNS (Hanson and Frey, 2008). The side effects and mortality rates for the clinical trials of FGF2 were high, and therefore, further experimental investigations are needed to assess the possibility to achieving a pharmacological therapeutic level in the brain, and also focusing on the potential mechanisms of FGF2 delivery outcomes in the pre-clinical trials can be strategy to help researchers determine when to administer treatment to stroke onset. No other studies comparing FGF2 with placebo in patients with acute stroke have been carried out since these studies.

Furthermore, studies were carried out on the association of FGF23 with stroke (Kendrick et al., 2011; Ix et al., 2012), but these studies were limited because they did not examine FGF23 association with other subtypes of ischemic stroke. Kendrick et al. conducted a study and found no significant association of FGF23 with stroke on 43 persons who had advanced chronic kidney disease (CKD) (Kendrick et al., 2011), whereas the study from Heart and Soul reported 36 individuals with higher FGF23 that was associated with higher risk of incident stroke (Parker et al., 2010). The Northern Manhattan Study (NOMAS) conducted on 212 patients also reported no association of FGF23 with ischemic stroke after adjusting the risk of stroke factors (Wright et al., 2014). However, Panwar et al. demonstrated that there was no significant association of FGF23 concentrations with all incident stroke, but in a prespecified analyses, only cardioembolic stroke occurred due to the high level of FGF23 (Panwar et al., 2015), which is consistent with "FGF23 association with ischemic stroke and its subtypes" study by Zheng et al. (Zheng et al., 2020). Nearly two decades passed, and more randomized clinical trials of FGF in patients with acute ischemic stroke are needed (Wahlgren and Ahmed, 2004). The new goal of treatment may be enhancing functional recovery rather than achieving immediate neuroprotection. Furthermore, artificial FGFR agonists may be useful alternatives to FGF for the treatment of ischemic vascular disease (Ballinger et al., 1999).

\section{Serum Biomarkers}

The use of blood biomarkers for stroke is being increasingly accepted since biomarkers might help neurologists evaluate stroke. Guo et al. demonstrated that the increase in FGF levels is maintained during the first 2 weeks after stroke (Guo et al., 2006). Previous studies have reported that serum FGF levels in patients with acute ischemic stroke are significantly higher than those of patients in the control group (Song et al., 2002; GolabJanowska et al., 2019). The prognostic value of FGF21 was conducted on patients with acute ischemic stroke. Proteomic analysis of 25 patients revealed that FGF 21 is expressed at lower levels in intracranial blood than in systemic arterial blood (Maglinger et al., 2020). The potential mechanism associated with FGF21 and acute ischemic stroke outcomes has not been fully elucidated due some limitations (Zheng et al., 2021). Firstly, there might be a selection bias and generalizability concern, since 
other race/ethnics were not part of the study. However, a balance for including and excluding patients based on the baseline characteristics from the China National Stroke Registry (Wang et al., 2011) was of a benefit, because the participants for the study were from China Antihypertensive Trial in Acute Ischemic Stroke (CATIS). Secondly, changes were not detected from the time of hospitalization because the FGF21 levels and blood pressure were taken once at baseline for the participants, resulting in no evidence of data to determine the association of FGF21 levels and acute ischemic stroke (Wang Z. et al., 2011). Lastly, individuals hospitalized for more than $24 \mathrm{~h}$ were included in this study which might be a possibility of inaccuracy of FGF21 levels from the onset of stroke, though a report showed that FGFs are still maintained for 3 days after ischemia (Wang et al., 2016).

The prognostic value of the mechanism of higher levels of FGF23 associated with the risk of stroke is still under exploration, but some contributions have been made to help understand its concept from a meta-analysis study. Studies showed that FGF23 has the ability to exert a direct toxic effect on the heart and vessels which could lead to the activation of renin-angiotensin-aldosterone due to the toxicity of FGF23 (Yao et al., 2018), and this could later induce the pathophysiology of stroke and hypertension (Ma et al., 2010). A clinical study revealed that the population with higher FGF23 concentrations have higher occurrence of left ventricle hypertrophy (LVH) (Mirza et al., 2009). Therefore, considering the relation of $\mathrm{LVH}$ with elevated arteriosclerosis, it could be partly explained that FGF23 has an effect on stroke risk. Also, the association of FGF23 with ischemic stroke and its subtypes has some limitations, because the methodologies used to measure the FGF23 levels could cause some biasness in the results (Zheng et al., 2020). Bioinformatics analysis of genes revealed that brain FGF9 gene expression levels are increased in stroke patients (Zou et al., 2019). However, a prospective study including 109 stroke patients did not reveal differences between FGF plasma levels at different time points. Therefore, more research on FGF levels in stroke patients and healthy people involving a larger number of samples should be is needed.

\section{CONCLUSION AND PROSPECT}

As presented in this review, FGFs could be used to treat stroke due to their pharmacological actions on multiple targets, including the ability to directly promote neuronal survival, enhance angiogenesis, protect against $\mathrm{BBB}$ disruption, regulate microglia, reduce the infarct size and promote neurological

\section{REFERENCES}

Arsava, E. M., Kim, G.-M., Oliveira-Filho, J., Gungor, L., Noh, H. J., Lordelo, M. d. J., et al. (2016). Prediction of Early Recurrence after Acute Ischemic Stroke. JAMA Neurol. 73 (2168-6157), 396-401. doi:10.1001/jamaneurol. 2015.4949

Asgharzade, S., Talaei, A., Farkhondeh, T., and Forouzanfar, F. (2020). Combining Growth Factor and Stem Cell Therapy for Stroke Rehabilitation, A Review. Cdt 21 (8), 781-791. doi:10.2174/ 1389450121666200107100747 function (Lin et al., 2018; Yang and Torbey, 2020). Biochemical studies have revealed the mechanisms by which FGFs improve neurological function after ischemic stroke.

The most studied FGFs to date are FGF1 and FGF2. There are some limitations to the application of FGF1, as it cannot cross the $\mathrm{BBB}$ to enter the brain and can lead to metastasis and tumorigenesis because of its mitogenic effect. These limitations could be limiting factors to the development of FGF2 as a protective agent affecting multiple targets for the treatment of stroke, and should be carefully considered. Combination therapies involving FGFs have been shown to exert therapeutic effects against stroke via multiple mechanisms in animal experiments but have not yet been applied clinically (Asgharzade et al., 2020).

In this review, we summarized the protective and survivalpromoting effects of FGFs in stroke models. Future research on FGFs and the development of FGFs as novel drugs to treat ischemic stroke are needed to improve clinical outcomes and develop a strategy for functional recovery. The safety, efficacy, timing and dose-dependent effects of FGF in animals and patients following stroke need to be determined in the future. Ongoing studies investigating FGF as a new drug target in the ischemic brain will provide novel insights into the role of FGF in the development of stroke pathogenesis and aid in the development of therapies to enhance stroke recovery.

\section{AUTHOR CONTRIBUTIONS}

LL and XW conceived this idea for the opinion and reviewed the manuscript. $\mathrm{CD}, \mathrm{KC}$, and $\mathrm{XW}$ were responsible for writing the paper, LL and WH organized the information and editing of the article. JC and JH were responsible for collecting the materials. All the authors contributed to critical scrutiny of the literature, and its approval.

\section{FUNDING}

This work was supported by the National Natural Science Foundation of China (No. 81771284, 81971180), and Natural Science Foundation of Zhejiang Province (LQ19H090012), Medical and Health Science and Technology Program of Zhejiang Province (WKJ-ZJ-2130), and CAMS Innovation Fund for Medical Sciences (2019-I2M-5-028).

Ay, H., Ay, I., Koroshetz, W., and Finklestein, S. (1999). Potential Usefulness of Basic Fibroblast Growth Factor as a Treatment for Stroke. Cerebrovasc. Dis. 9, 131-135. doi:10.1159/000015941

Ballinger, M. D., Shyamala, V., Forrest, L. D., Deuter-Reinhard, M., Doyle, L. V., Wang, J.-x., et al. (1999). Semirational Design of a Potent, Artificial Agonist of Fibroblast Growth Factor Receptors. Nat. Biotechnol. 17 (12), 1199-1204. doi:10.1038/70746

Bao, X., Wei, J., Feng, M., Lu, S., Li, G., Dou, W., et al. (2011). Transplantation of Human Bone Marrow-Derived Mesenchymal Stem Cells Promotes Behavioral Recovery and Endogenous Neurogenesis after Cerebral Ischemia in Rats. Brain Res. 1367, 103-113. doi:10.1016/j.brainres.2010.10.063 
Beck, H., and Plate, K. H. (2009). Angiogenesis after Cerebral Ischemia. Acta Neuropathol. 117, 481-496. doi:10.1007/s00401-009-0483-6

Beenken, A., and Mohammadi, M. (2009). The FGF Family: Biology, Pathophysiology and Therapy. Nat. Rev. Drug Discov. 8, 235-253. doi:10. 1038/nrd2792

Belov, A. A., and Mohammadi, M. (2013). Molecular Mechanisms of Fibroblast Growth Factor Signaling in Physiology and Pathology. Cold Spring Harbor Perspect. Biol. 5, a015958. doi:10.1101/cshperspect.a015958

Beyer, T., Werner, S., Dickson, C., and Grose, R. (2003). Fibroblast Growth Factor 22 and its Potential Role during Skin Development and Repair. Exp. Cel Res. 287, 228-236. doi:10.1016/s0014-4827(03)00139-3

Bogousslavsky, J., Salinas, E. O., Pallay, A., Donnan, G. A., Fieschi, C., Kaste, M., et al. (2002). Fiblast (Trafermin) in Acute Stroke: Results of the EuropeanAustralian Phase II/III Safety and Efficacy Trial. Cerebrovasc. Dis. 14 (3-4), 239-251. doi:10.1159/000065683

Bonsack, B., Corey, S., Shear, A., Heyck, M., Cozene, B., Sadanandan, N., et al. (2020). Mesenchymal Stem Cell Therapy Alleviates the Neuroinflammation Associated with Acquired Brain Injury. CNS Neurosci. Ther. 26 (6), 603-615. doi:10.1111/cns.13378

Boshoff, E. L., Fletcher, E. J. R., and Duty, S. (2018). Fibroblast Growth Factor 20 Is Protective towards Dopaminergic Neurons In Vivo in a Paracrine Manner. Neuropharmacology 137, 156-163. doi:10.1016/j.neuropharm.2018.04.017

Campbell, B. C. V., and Khatri, P. (2020). Stroke. Lancet 396 (10244), 129-142. doi:10.1016/s0140-6736(20)31179-x

Candelario-Jalil, E., and Paul, S. (2021). Impact of Aging and Comorbidities on Ischemic Stroke Outcomes in Preclinical Animal Models: A Translational Perspective. Exp. Neurol. 335, 113494. doi:10.1016/j.expneurol.2020. 113494

Causes of Death Collaborators (2017). Global, Regional, and National Age-Sex Specific Mortality for 264 Causes of Death, 1980-2016: a Systematic Analysis for the Global Burden of Disease Study 2016. Lancet 390, 1151-1210. doi:10.1016/ S0140-6736(17)32152-9

Charoenlarp, P., Rajendran, A. K., and Iseki, S. (2017). Role of Fibroblast Growth Factors in Bone Regeneration. Inflamm. Regener 37, 10. doi:10.1186/s41232017-0043-8

Chau, M. D. L., Gao, J., Yang, Q., Wu, Z., and Gromada, J. (2010). Fibroblast Growth Factor 21 Regulates Energy Metabolism by Activating the AMPKSIRT1-PGC-1a Pathway. Proc. Natl. Acad. Sci. USA 107 (28), 12553-12558. doi:10.1073/pnas.1006962107

Chen, J., Hu, J., Liu, H., Xiong, Y., Zou, Y., Huang, W., et al. (2018). FGF21 Protects the Blood-Brain Barrier by Upregulating PPAR $\gamma$ via FGFR1/ $\beta$-Klotho after Traumatic Brain Injury. J. Neurotrauma 35 (17), 2091-2103. doi:10.1089/neu. 2017.5271

Chen, J., Wang, X., Hu, J., Du, J., Dordoe, C., Zhou, Q., et al. (2020). FGF20 Protected against BBB Disruption after Traumatic Brain Injury by Upregulating Junction Protein Expression and Inhibiting the Inflammatory Response. Front. Pharmacol. 11, 590669. doi:10.3389/fphar.2020.590669

Chen, J., Zhang, X., Liu, X., Zhang, C., Shang, W., Xue, J., et al. (2019). Ginsenoside Rg1 Promotes Cerebral Angiogenesis via the PI3K/Akt/mTOR Signaling Pathway in Ischemic Mice. Eur. J. Pharmacol. 856, 172418. doi:10.1016/j. ejphar.2019.172418

Cheng, X., Wang, Z., Yang, J., Ma, M., Lu, T., Xu, G., et al. (2011). Acidic Fibroblast Growth Factor Delivered Intranasally Induces Neurogenesis and Angiogenesis in Rats after Ischemic Stroke. Neurol. Res. 33, 675-680. doi:10.1179/ 1743132810Y.0000000004

Clark, W. M., Schim, J. D., Kasner, S. E., and Victor, S. (2000). Trafermin in Acute Ischemic Stroke. Results a phase II/III randomized efficacy Study Neurol. 54, A88. doi:10.1159/000065683

Coban, M., Inci, A., Yllmaz, U., and Asilturk, E. (2018). The Association of Fibroblast Growth Factor 23 with Arterial Stiffness and Atherosclerosis in Patients with Autosomal Dominant Polycystic Kidney Disease. Kidney Blood Press. Res. 43 (1423-0143), 1160-1173. doi:10.1159/000492244

Cui, Y., and Li, Q. (2013). Expression and Functions of Fibroblast Growth Factor 10 in the Mouse Mammary Gland. Ijms 14, 4094-4105. doi:10.3390/ ijms14024094

de Lapeyriere, O., Rosnet, O., Benharroch, D., Raybaud, F., Marchetto, S., Planche, J., et al. (1990). Structure, Chromosome Mapping and Expression of the Murine Fgf-6 Gene. Oncogene 5, 823-831.
Dickson, C., Deed, R., Dixon, M., and Peters, G. (1989). The Structure and Function of the Int-2 Oncogene. Prog. Growth Factor. Res. 1, 123-132. doi:10.1016/0955-2235(89)90006-9

Dutchak, P. A., Katafuchi, T., Bookout, A. L., Choi, J. H., Yu, R. T., Mangelsdorf, D. J., et al. (2012). Fibroblast Growth Factor-21 Regulates PPAR $\gamma$ Activity and the Antidiabetic Actions of Thiazolidinediones. Cell 148, 556-567. doi:10.1016/j. cell.2011.11.062

Ellsworth, J. L., Garcia, R., Yu, J., and Kindy, M. S. (2003). Fibroblast Growth Factor-18 Reduced Infarct Volumes and Behavioral Deficits after Transient Occlusion of the Middle Cerebral Artery in Rats. Stroke 34 (6), 1507-1512. doi:10.1161/01.STR.0000071760.66720.5F

Ellsworth, J. L., Garcia, R., Yu, J., and Kindy, M. S. (2004). Time Window of Fibroblast Growth Factor-18-Mediated Neuroprotection after Occlusion of the Middle Cerebral Artery in Rats*. J. Cereb. Blood Flow Metab. 24 (1), 114-123. doi:10.1097/01.WCB.0000100063.36077.CD

Fandler-Höfler, S., Enzinger, C., Kneihsl, M., Pinter, D., Eppinger, S., ObermayerPietsch, B., et al. (2019). Early Renal Dysfunction and Fibroblast Growth Factor-23 in Patients with Small Vessel Disease-Related Stroke. Sci. Rep. 9 (1), 15410. doi:10.1038/s41598-019-51965-5

Finch, P. W., and Rubin, J. S. (2004). Keratinocyte Growth Factor/Fibroblast Growth Factor 7, a Homeostatic Factor with Therapeutic Potential for Epithelial Protection and Repair. Adv. Cancer Res. 91, 69-136. doi:10.1016/ S0065-230X(04)91003-2

Fleury, C., Bernard, M., and Vayssière, J. L. (2002). Mitochondrial Reactive Oxygen Species in Cell Death Signaling. Biochimie 84 (2-3), 131-141. doi:10.1016/ s0300-9084(02)01369-x

Freret, T., Chazalviel, L., Roussel, S., Bernaudin, M., Schumann-Bard, P., and Boulouard, M. (2006). Long-term Functional Outcome Following Transient Middle Cerebral Artery Occlusion in the Rat: Correlation between Brain Damage and Behavioral Impairment. Behav. Neurosci. 120 (6), 1285-1298. doi:10.1037/0735-7044.120.6.1285

Golab-Janowska, M., Paczkowska, E., Machalinski, B., Kotlega, D., Meller, A., Safranow, K., et al. (2019). Elevated Inflammatory Parameter Levels Negatively Impact Populations of Circulating Stem Cells (CD133+), Early Endothelial Progenitor Cells (CD133+/VEGFR2+), and Fibroblast Growth Factor in Stroke Patients. Curr. Neurovasc. Res. 16 (1), 19-26. doi:10.2174/ 1567202616666190129164906

Goldfarb, M. (2005). Fibroblast Growth Factor Homologous Factors: Evolution, Structure, and Function. Cytokine Growth Factor. Rev. 16, 215-220. doi:10. 1016/j.cytogfr.2005.02.002

Gospodarowicz, D. (1975). Purification of a Fibroblast Growth Factor from Bovine Pituitary. J. Biol. Chem. 250, 2515-2520. doi:10.1016/S0021-9258(19)41631-1

Griessenauer, C. J., Medin, C., Maingard, J., Chandra, R. V., Ng, W., Brooks, D. M., et al. (2018). Endovascular Mechanical Thrombectomy in Large-Vessel Occlusion Ischemic Stroke Presenting with Low National Institutes of Health Stroke Scale: Systematic Review and Meta-Analysis. World Neurosurg. 110, 263-269. doi:10.1016/j.wneu.2017.11.076

Guo, H., Li, H., Cheng, M., Jin, X., Zhao, Y., and Yi, M. (2006). Serial Measurement of Serum Basic Fibroblast Growth Factor in Patients with Acute Cerebral Infarction. Neurosci. Lett. 393 (1), 56-59. doi:10.1016/j.neulet.2005.09.043

Hanson, L. R., and Frey, W. H. (2008). Intranasal Delivery Bypasses the BloodBrain Barrier to Target Therapeutic Agents to the Central Nervous System and Treat Neurodegenerative Disease. BMC Neurosci. 9 (Suppl. 3). doi:10.1186/ 1471-2202-9-S3-S5

Hoshikawa, M., Yonamine, A., Konishi, M., and Itoh, N. (2002). FGF-18 Is a Neuron-Derived Glial Cell Growth Factor Expressed in the Rat Brain during Early Postnatal Development. Mol. Brain Res. 105, 60-66. doi:10.1016/s0169$328 \mathrm{x}(02) 00393-5$

Huang, M., Williams, J., Kong, B., Zhu, Y., Li, G., Zhu, Z., et al. (2018). Fibroblast Growth Factor 15 Deficiency Increases Susceptibility but Does Not Improve Repair to Acetaminophen-Induced Liver Injury in Mice. Dig. Liver Dis. 50, 175-180. doi:10.1016/j.dld.2017.08.023

Hutter-Schmid, B., and Humpel, C. (2016). Platelet-derived Growth Factor Receptor-Beta Is Differentially Regulated in Primary Mouse Pericytes and Brain Slices. Curr. Neurovasc. Res. 13 (2), 127-134. doi:10.2174/ 156720261366616021912041

Ikeda, N., Nonoguchi, N., Zhao, M. Z., Watanabe, T., Kajimoto, Y., Furutama, D., et al. (2005). Bone Marrow Stromal Cells that Enhanced Fibroblast Growth 
Factor-2 Secretion by Herpes Simplex Virus Vector Improve Neurological Outcome after Transient Focal Cerebral Ischemia in Rats. Stroke 36, 2725-2730. doi:10.1161/01.STR.0000190006.88896.d3

Inagaki, T., Dutchak, P., Zhao, G., Ding, X., Gautron, L., Parameswara, V., et al. (2007). Endocrine Regulation of the Fasting Response by PPARalpha-Mediated Induction of Fibroblast Growth Factor 21. Cell Metab 5, 415-425. doi:10.1016/j. cmet.2007.05.003

Itoh, N., and Ornitz, D. M. (2011). Fibroblast Growth Factors: from Molecular Evolution to Roles in Development, Metabolism and Disease. J. Biochem. 149 (2), 121-130. doi:10.1093/jb/mvq121

Iwasawa, E., Ishibashi, S., Suzuki, M., Li, F., Ichijo, M., Miki, K., et al. (2018). Sphingosine-1-Phosphate Receptor 1 Activation Enhances Leptomeningeal Collateral Development and Improves Outcome after Stroke in Mice. J. Stroke Cerebrovasc. Dis. 27 (5), 1237-1251. doi:10.1016/j. jstrokecerebrovasdis.2017.11.040

Ix, J. H., Katz, R., Kestenbaum, B. R., de Boer, I. H., Chonchol, M., Mukamal, K. J., et al. (2012). Fibroblast Growth Factor-23 and Death, Heart Failure, and Cardiovascular Events in Community-Living Individuals: CHS (Cardiovascular Health Study). J. Am. Coll. Cardiol. 60, 200-207. doi:10. 1016/j.jacc.2012.03.040

Jacques, T. S., Skepper, J. N., and Navaratnam, V. (1999). Fibroblast Growth Factor-1 Improves the Survival and Regeneration of Rat Vagal Preganglionic Neurons Following Axon Injury. Neurosci. Lett. 276, 197-200. doi:10.1016/ s0304-3940(99)00832-0

Jiang, M., Gong, D., and Fan, Y. (2016). Elevated Fibroblast Growth Factor-23 and Risk of Cardiovascular Disease or Mortality in the General Population: A MetaAnalysis. Int. J. Cardiol. 222, 342-345. doi:10.1016/j.ijcard.2016.07.276

Jiang, X., Andjelkovic, A. V., Zhu, L., Yang, T., Bennett, M. V. L., Chen, J., et al. (2018). Blood-brain Barrier Dysfunction and Recovery after Ischemic Stroke. Prog. Neurobiol. 163-164, 144-171. doi:10.1016/j.pneurobio.2017. 10.001

Jiang, Y., Lin, L., Liu, N., Wang, Q., Yuan, J., Li, Y., et al. (2020). FGF21 Protects against Aggravated Blood-Brain Barrier Disruption after Ischemic Focal Stroke in Diabetic Db/db Male Mice via Cerebrovascular PPARgamma Activation. Int. J. Mol. Sci. 21 (3). doi:10.3390/ijms21030824

Kanda, T., Iwasaki, T., Nakamura, S., Kurokawa, T., Ikeda, K., and Mizusawa, H. (2000). Self-secretion of Fibroblast Growth Factor-9 Supports Basal Forebrain Cholinergic Neurons in an Autocrine/paracrine Manner. Brain Res. 876, 22-30. doi:10.1016/s0006-8993(00)02563-4

Kawamata, T., Dietrich, W. D., Schallert, T., Gotts, J. E., Cocke, R. R., Benowitz, L. I., et al. (1997). Intracisternal Basic Fibroblast Growth Factor Enhances Functional Recovery and Up-Regulates the Expression of a Molecular Marker of Neuronal Sprouting Following Focal Cerebral Infarction. Proc. Natl. Acad. Sci. 94 (15), 8179-8184. doi:10.1073/pnas.94.15.8179

Kelly, A. G., and Holloway, R. G. (2018). Guideline: The AHA/ASA Made 217 Recommendations for Early Management of Acute Ischemic Stroke in Adults. Ann. Intern. Med. 168 (12), JC63. doi:10.7326/ACPJC-2018-168-12-063

Kendrick, J., Cheung, A. K., Kaufman, J. S., Greene, T., Roberts, W. L., Smits, G., et al. (2011). FGF-23 Associates with Death, Cardiovascular Events, and Initiation of Chronic Dialysis. J. Am. Soc. Nephrol. 22 (10), 1913-1922. doi:10.1681/ASN.2010121224

Konishi, M., Mikami, T., Yamasaki, M., Miyake, A., and Itoh, N. (2000). Fibroblast Growth Factor-16 Is a Growth Factor for Embryonic Brown Adipocytes. J. Biol. Chem. 275, 12119-12122. doi:10.1074/jbc.275.16.12119

Koo, H. Y., El-Baz, L. M. F., House, S. L., Cilvik, S. N., Dorry, S. J., Shoukry, N. M., et al. (2018). Fibroblast Growth Factor 2 Decreases Bleomycin-Induced Pulmonary Fibrosis and Inhibits Fibroblast Collagen Production and Myofibroblast Differentiation. J. Pathol. 246 (1), 54-66. doi:10.1002/path. 5106

Krejci, P., Prochazkova, J., Bryja, V., Kozubik, A., and Wilcox, W. R. (2009). Molecular Pathology of the Fibroblast Growth Factor Family. Hum. Mutat. 30, 1245-1255. doi:10.1002/humu.21067

Lanfranconi, S., Locatelli, F., Corti, S., Candelise, L., Comi, G. P., Baron, P. L., et al. (2011). Growth Factors in Ischemic Stroke. J. Cel Mol. Med. 15 (8), 1645-1687. doi:10.1111/j.1582-4934.2009.00987.x

Lee, K. W., Yim, H. S., Shin, J., Lee, C., Lee, J. H., and Jeong, J. Y. (2017). FGF11 Induced by Hypoxia Interacts with HIF-1alpha and Enhances its Stability. FEBS Lett. 591, 348-357. doi:10.1002/1873-3468.12547
Leng, Y., Wang, Z., Tsai, L. K., Leeds, P., Fessler, E. B., Wang, J., et al. (2015). FGF21, a Novel Metabolic Regulator, Has a Robust Neuroprotective Role and Is Markedly Elevated in Neurons by Mood Stabilizers. Mol. Psychiatry 20, 215-223. doi:10.1038/mp.2013.192

Li, H., Zhou, X., Li, Y., Ma, X., Gonzales, R. J., Qiu, S., et al. (2019). The Selective Sphingosine 1-phosphate Receptor 1 Modulator RP101075 Improves Microvascular Circulation after Cerebrovascular Thrombosis. FASEB J. 33 (10), 10935-10941. doi:10.1096/fj.201900282R

Li, Q., and Stephenson, D. (2002). Postischemic Administration of Basic Fibroblast Growth Factor Improves Sensorimotor Function and Reduces Infarct Size Following Permanent Focal Cerebral Ischemia in the Rat. Exp. Neurol. 177, 531-537. doi:10.1006/exnr.2002.7994

Li, S., Lu, Y., Ding, D., Ma, Z., Xing, X., Hua, X., et al. (2020). Fibroblast Growth Factor 2 Contributes to the Effect of Salidroside on Dendritic and Synaptic Plasticity after Cerebral Ischemia/reperfusion Injury. Aging 12 (11), 10951-10968. doi:10.18632/aging.103308

Li, Y. H., Fu, H. L., Tian, M. L., Wang, Y. Q., Chen, W., Cai, L. L., et al. (2016). Neuron-derived FGF10 Ameliorates Cerebral Ischemia Injury via Inhibiting NF-Kappa B-dependent Neuroinflammation and Activating PI3K/Akt Survival Signaling Pathway in Mice. Sci. Rep. 6, 19869. doi:10.1038/srep19869

Li, Y. H., Yang, L. Y., Chen, W., Li, Y. K., and Yuan, H. B. (2015). Fibroblast Growth Factor 10 Protects Neuron against Oxygen-Glucose Deprivation Injury through Inducing Heme Oxygenase-1. Biochem. Biophys. Res. Commun. 456 (1), 225-231. doi:10.1016/j.bbrc.2014.11.063

Lim, M. S., Lee, S. Y., and Park, C. H. (2015). FGF8 Is Essential for Functionality of Induced Neural Precursor Cell-Derived Dopaminergic Neurons. Int. J. Stem Cell 8, 228-234. doi:10.15283/ijsc.2015.8.2.228

Lin, L., Wang, Q., Qian, K., Cao, Z., Xiao, J., Wang, X., et al. (2018). bFGF Protects against Oxygen Glucose Deprivation/Reoxygenation-Induced Endothelial Monolayer Permeability via S1PR1-Dependent Mechanisms. Mol. Neurobiol. 55 (4), 3131-3142. doi:10.1007/s12035-017-0544-0

Lin, Z., Pan, X., Wu, F., Ye, D., Zhang, Y., Wang, Y., et al. (2015). Fibroblast Growth Factor 21 Prevents Atherosclerosis by Suppression of Hepatic Sterol Regulatory Element-Binding Protein-2 and Induction of Adiponectin in Mice. Circulation 131, 1861-1871. doi:10.1161/CIRCULATIONAHA.115.015308

Lindholm, D., Harikka, J., da Penha Berzaghi, M., Castren, E., Tzimagiorgis, G., Hughes, R. A., et al. (1994). Fibroblast Growth Factor-5 Promotes Differentiation of Cultured Rat Septal Cholinergic and Raphe Serotonergic Neurons: Comparison with the Effects of Neurotrophins. Eur. J. Neurosci. 6 (2), 244-252.

Liu, J., Zhou, Cx., Zhang, Z., Wang, L., Jing, Z., and Wang, Z. (2012). Synergistic Mechanism of Gene Expression and Pathways between Jasminoidin and Ursodeoxycholic Acid in Treating Focal Cerebral Ischemia-Reperfusion Injury. CNS Neurosci. Ther. 18 (8), 674-682. doi:10.1111/j.1755-5949.2012. 00348.x

Lo, E. H., Dalkara, T., and Moskowitz, M. A. (2003). Mechanisms, Challenges and Opportunities in Stroke. Nat. Rev. Neurosci. 4 (5), 399-414. doi:10.1038/ nrn1106

Lu, H., Shi, X., Wu, G., Zhu, J., Song, C., Zhang, Q., et al. (2015). FGF13 Regulates Proliferation and Differentiation of Skeletal Muscle by Down-Regulating Spryl. Cell Prolif 48, 550-560. doi:10.1111/cpr.12200

Lum, M., Turbic, A., Mitrovic, B., and Turnley, A. M. (2009). Fibroblast Growth Factor-9 Inhibits Astrocyte Differentiation of Adult Mouse Neural Progenitor Cells. J. Neurosci. Res. 87, 2201-2210. doi:10.1002/jnr.22047

Ma, T. K., Kam, K. K., Yan, B. P., and Lam, Y. Y. (2010). Renin-angiotensinaldosterone System Blockade for Cardiovascular Diseases: Current Status. $\mathrm{Br}$. J. Pharmacol. 160 (6), 1273-1292. doi:10.1111/j.1476-5381.2010.00750.x

Maglinger, B., Frank, J. A., McLouth, C. J., Trout, A. L., Roberts, J. M., Grupke, S., et al. (20202020-01611). Proteomic Changes in Intracranial Blood during Human Ischemic Stroke. J. Neurointer. Surg. 13 (4), 395-399. doi:10.1136/ neurintsurg-2020-016118

Maruoka, Y., Ohbayashi, N., Hoshikawa, M., Itoh, N., Hogan, B. L., and Furuta, Y. (1998). Comparison of the Expression of Three Highly Related Genes, Fgf8, Fgf17 and Fgf18, in the Mouse Embryo. Mech. Dev. 74, 175-177. doi:10.1016/ s0925-4773(98)00061-6

Matkar, P. N., Ariyagunarajah, R., Leong-Poi, H., and Singh, K. K. (2017). Friends Turned Foes: Angiogenic Growth Factors beyond Angiogenesis. Biomolecules 7 (4), 74. doi:10.3390/biom7040074 
Mirza, M. A., Larsson, A., Melhus, H., Lind, L., and Larsson, T. E. (2009). Serum Intact FGF23 Associate with Left Ventricular Mass, Hypertrophy and Geometry in an Elderly Population. Atherosclerosis 207 (2), 546-551. doi:10. 1016/j.atherosclerosis.2009.05.013

Mudo, G., Bonomo, A., Di Liberto, V., Frinchi, M., Fuxe, K., and Belluardo, N. (2009). The FGF-2/FGFRs Neurotrophic System Promotes Neurogenesis in the Adult Brain. J. Neural Transm. 116, 995-1005. doi:10.1007/s00702-0090207-z

Nakamura, K., Arimura, K., Nishimura, A., Tachibana, M., Yoshikawa, Y., Makihara, N., et al. (2016). Possible Involvement of Basic FGF in the Upregulation of PDGFRbeta in Pericytes after Ischemic Stroke. Brain Res. 1630, 98-108. doi:10.1016/j.brainres.2015.11.003

Nishimura, T., Nakatake, Y., Konishi, M., and Itoh, N. (2000). Identification of a Novel FGF, FGF-21, Preferentially Expressed in the Liver. Biochim. Biophys. Acta 1492, 203-206. doi:10.1016/s0167-4781(00)00067-1

Nishimura, T., Utsunomiya, Y., Hoshikawa, M., Ohuchi, H., and Itoh, N. (1999). Structure and Expression of a Novel Human FGF, FGF-19, Expressed in the Fetal Brain. Biochim. Biophys. Acta 1444, 148-151. doi:10.1016/s0167-4781(98) 00255-3

Ohbayashi, N., Hoshikawa, M., Kimura, S., Yamasaki, M., Fukui, S., and Itoh, N. (1998). Structure and Expression of the mRNA Encoding a Novel Fibroblast Growth Factor, FGF-18. J. Biol. Chem. 273, 18161-18164. doi:10.1074/jbc.273. 29.18161

Okunieff, P., Fenton, B. M., Zhang, L., Kern, F. G., Wu, T., Greg, J. R., et al. (2003). Fibroblast Growth Factors (FGFS) Increase Breast Tumor Growth Rate, Metastases, Blood Flow, and Oxygenation without Significant Change in Vascular Density. Adv. Exp. Med. Biol. 530, 593-601. doi:10.1007/978-14615-0075-9_58

Ornitz, D. M., and Itoh, N. (2001). Fibroblast Growth Factors. Genome Biol. 2, REVIEWS3005. doi:10.1186/gb-2001-2-3-reviews3005

Ornitz, D. M., and Itoh, N. (2015). The Fibroblast Growth Factor Signaling Pathway. Wiley Interdiscip. Rev. Dev. Biol. 4 (3), 215-266. doi:10.1002/ wdev.176

Panwar, B., Jenny, N. S., Howard, V. J., Wadley, V. G., Muntner, P., Kissela, B. M., et al. (2015). Fibroblast Growth Factor 23 and Risk of Incident Stroke in Community-Living Adults. Stroke 46 (2), 322-328. doi:10.1161/STROKEAHA. 114.007489

Parker, B. D., Schurgers, L. J., Brandenburg, V. M., Christenson, R. H., Vermeer, C., Ketteler, M., et al. (2010). The Associations of Fibroblast Growth Factor 23 and Uncarboxylated Matrix Gla Protein with Mortality in Coronary Artery Disease: the Heart and Soul Study. Ann. Intern. Med. 152 (10), 640-648. doi:10.7326/ 0003-4819-152-10-201005180-00004

Polnaszek, N., Kwabi-Addo, B., Wang, J., and Ittmann, M. (2004). FGF17 Is an Autocrine Prostatic Epithelial Growth Factor and Is Upregulated in Benign Prostatic Hyperplasia. Prostate 60, 18-24. doi:10.1002/pros.20026

Potthoff, M. J., Boney-Montoya, J., Choi, M., He, T., Sunny, N. E., Satapati, S., et al. (2011). FGF15/19 Regulates Hepatic Glucose Metabolism by Inhibiting the CREB-PGC-1alpha Pathway. Cel Metab 13, 729-738. doi:10.1016/j.cmet.2011. 03.019

Prabhakaran, S., Ruff, I., and Bernstein, R. A. (2015). Acute Stroke Intervention: a Systematic Review. JAMA 1538-3598, 1451-1462. doi:10.1001/jama.2015.3058

Rahman, A. A., Amruta, N., Pinteaux, E., and Bix, G. J. (2021). Neurogenesis after Stroke: A Therapeutic Perspective. Transl. Stroke Res. 12 (1), 1-14. doi:10.1007/ s12975-020-00841-w

Reuss, B., Dono, R., and Unsicker, K. (2003). Functions of Fibroblast Growth Factor (FGF)-2 and FGF-5 in Astroglial Differentiation and Blood-Brain Barrier Permeability: Evidence from Mouse Mutants. J. Neurosci. 23, 6404-6412. doi:10.1523/JNEUROSCI.23-16-06404.2003

Revest, J. M., DeMoerlooze, L., and Dickson, C. (2000). Fibroblast Growth Factor 9 Secretion Is Mediated by a Non-cleaved Amino-Terminal Signal Sequence. J. Biol. Chem. 275, 8083-8090. doi:10.1074/jbc.275.11.8083

Robertson, K. E., Tickle, C., and Darling, S. M. (1997). Shh, Fgf4 and Hoxd Gene Expression in the Mouse Limb Mutant Hypodactyly. Int. J. Dev. Biol. 41, 733-736.

Sadohara, T., Sugahara, K., Urashima, Y., Terasaki, H., and Lyama, K. (2001). Keratinocyte Growth Factor Prevents Ischemia-Induced Delayed Neuronal Death in the Hippocampal CA1 Field of the Gerbil Brain. Neuroreport 12 (1), 71-76. doi:10.1097/00001756-200101220-00022
Shakkottai, V. G., Xiao, M., Xu, L., Wong, M., Nerbonne, J. M., Ornitz, D. M., et al. (2009). FGF14 Regulates the Intrinsic Excitability of Cerebellar Purkinje Neurons. Neurobiol. Dis. 33, 81-88. doi:10.1016/j.nbd.2008.09.019

Shibahara, T., Ago, T., Nakamura, K., Tachibana, M., Yoshikawa, Y., Komori, M., et al. (2020). Pericyte-Mediated Tissue Repair through PDGFRbeta Promotes Peri-Infarct Astrogliosis, Oligodendrogenesis, and Functional Recovery after Acute Ischemic Stroke. eNeuro 7 (2). doi:10.1523/ENEURO.0474-19.2020

Song, S., Zheng, X., Wen, S., Huang, J., and Ding, D. (2002). Change of Serum Soluble Intercellular Adhesion Molecule and Basic Fibroblast Growth Factor in Patients with Acute Cerebral Infarction and its Clinical Significance. Zhonghua Yi Xue Za Zhi 82 (21), 1447-1449.

Stinear, C. M., Lang, C. E., Zeiler, S., and Byblow, W. D. (2020). Advances and Challenges in Stroke Rehabilitation. Lancet Neurol. 19 (4), 348-360. doi:10. 1016/S1474-4422(19)30415-6

Sun, D., Bullock, M. R., McGinn, M. J., Zhou, Z., Altememi, N., Hagood, S., et al. (2009). Basic Fibroblast Growth Factor-Enhanced Neurogenesis Contributes to Cognitive Recovery in Rats Following Traumatic Brain Injury. Exp. Neurol. 216, 56-65. doi:10.1016/j.expneurol.2008.11.011

Taylor, E. N., Rimm, E. B., Stampfer, M. J., and Curhan, G. C. (2011). Plasma Fibroblast Growth Factor 23, Parathyroid Hormone, Phosphorus, and Risk of Coronary Heart Disease. Am. Heart J. 161, 956-962. doi:10.1016/j.ahj.2011. 02.012

Tsai, M. J., Tsai, S. K., Huang, M. C., Liou, D. Y., Huang, S. L., Hsieh, W. H., et al. (2015). Acidic FGF Promotes Neurite Outgrowth of Cortical Neurons and Improves Neuroprotective Effect in a Cerebral Ischemic Rat Model. Neuroscience 305, 238-247. doi:10.1016/j.neuroscience.2015.07.074

van Venrooij, N. A., Pereira, R. C., Tintut, Y., Fishbein, M. C., Tumber, N., Demer, L. L., et al. (2014). FGF23 Protein Expression in Coronary Arteries Is Associated with Impaired Kidney Function. Nephrol. Dial. Transpl. 29, 1525-1532. doi:10. 1093/ndt/gft523

Wada, K., Sugimori, H., Bhide, P. G., Moskowitz, M. A., and Finklestein, S. P. (2003). Effect of Basic Fibroblast Growth Factor Treatment on Brain Progenitor Cells after Permanent Focal Ischemia in Rats. Stroke 34, 2722-2728. doi:10. 1161/01.STR.0000094421.61917.71

Wahlgren, N. G., and Ahmed, N. (2004). Neuroprotection in Cerebral Ischaemia: Facts and Fancies-Tthe Need for New Approaches. Cerebrovasc. Dis. 17, 153-166. doi:10.1159/000074808

Wan, H., Yang, Y., Li, M., Liu, X., Sun, Y., Wang, K., et al. (2019). Activation of AK005401 Aggravates Acute Ischemia/reperfusion Mediated Hippocampal Injury by Directly Targeting YY1/FGF21. Aging 11 (14), 5108-5123. doi:10. 18632/aging.102106

Wang, D., Liu, F., Zhu, L., Lin, P., Han, F., Wang, X., et al. (2020). FGF21 Alleviates Neuroinflammation Following Ischemic Stroke by Modulating the Temporal and Spatial Dynamics of Microglia/macrophages. J. Neuroinflammation 17 (1), 257. doi:10.1186/s12974-020-01921-2

Wang, H. W., Jiang, X., Zhang, Y., Wang, J., Xie, J., Wang, Y. Q., et al. (2019). FGF21 Protects against Hypoxia Injury through Inducing HSP72 in Cerebral Microvascular Endothelial Cells. Front. Pharmacol. 10, 101. doi:10.3389/fphar. 2019.00101

Wang, Q., Yuan, J., Yu, Z., Lin, L., Jiang, Y., Cao, Z., et al. (2018). FGF21 Attenuates High-Fat Diet-Induced Cognitive Impairment via Metabolic Regulation and Anti-inflammation of Obese Mice. Mol. Neurobiol. 55, 4702-4717. doi:10.1007/ s12035-017-0663-7

Wang, X. M., Xiao, H., Liu, L. L., Cheng, D., Li, X. J., and Si, L. Y. (2016). FGF21 Represses Cerebrovascular Aging via Improving Mitochondrial Biogenesis and Inhibiting P53 Signaling Pathway in an AMPK-dependent Manner. Exp. Cel Res. 346 (2), 147-156. doi:10.1016/j.yexcr.2016.06.020

Wang, Y., Liao, X., Zhao, X., Wang, D. Z., Wang, C., Nguyen-Huynh, M. N., et al. (2011). Using Recombinant Tissue Plasminogen Activator to Treat Acute Ischemic Stroke in China: Analysis of the Results from the Chinese National Stroke Registry (CNSR). Stroke 42 (6), 1658-1664. doi:10.1161/ STROKEAHA.110.604249

Wang, Z. G., Cheng, Y., Yu, X. C., Ye, L. B., Xia, Q. H., Johnson, N. R., et al. (2016). bFGF Protects against Blood-Brain Barrier Damage through Junction Protein Regulation via PI3K-Akt-Rac1 Pathway Following Traumatic Brain Injury. Mol. Neurobiol. 53, 7298-7311. doi:10.1007/s12035-015-9583-6

Wang, Z., Leng, Y., Wang, J., Liao, H. M., Bergman, J., Leeds, P., et al. (2016). Tubastatin A, an HDAC6 Inhibitor, Alleviates Stroke-Induced Brain Infarction 
and Functional Deficits: Potential Roles of Alpha-Tubulin Acetylation and FGF-21 Up-Regulation. Sci. Rep. 6, 19626. doi:10.1038/srep19626

Watanabe, M., Singhal, G., Fisher, F. M., Beck, T. C., Morgan, D. A., Socciarelli, F., et al. (2020). Liver-derived FGF21 Is Essential for Full Adaptation to Ketogenic Diet but Does Not Regulate Glucose Homeostasis. Endocrine 67 (1), 95-108. doi:10.1007/s12020-019-02124-3

Woodbury, M. E., and Ikezu, T. (2014). Fibroblast Growth Factor-2 Signaling in Neurogenesis and Neurodegeneration. J. Neuroimmune Pharmacol. 9 (2), 92-101. doi:10.1007/s11481-013-9501-5

Wright, C. B., Dong, C., Stark, M., Silverberg, S., Rundek, T., Elkind, M., S., et al. (2014). Plasma FGF23 and the Risk of Stroke: The Northern Manhattan Study (NOMAS). Neurology 82, 1700-1706. doi:10.1212/WNL.0000000000000410

Wu, F., Chen, Z., Tang, C., Zhang, J., Cheng, L., Zuo, H., et al. (2017). Acid Fibroblast Growth Factor Preserves Blood-Brain Barrier Integrity by Activating the PI3K-Akt-Racl Pathway and Inhibiting RhoA Following Traumatic Brain Injury. Am. J. Transl. Res. 9 (3), 910-925.

Wu, L., Qian, L., Zhang, L., Zhang, J., Zhou, J., Li, Y., et al. (2020). Fibroblast Growth Factor 21 Is Related to Atherosclerosis Independent of Nonalcoholic Fatty Liver Disease and Predicts Atherosclerotic Cardiovascular Events. J. Am. Heart Assoc. 9 (11), e015226. doi:10.1161/JAHA.119.015226

Xu, H., Li, X. K., Zheng, Q., Huang, Y. D., Yao, C. C., Su, Z. J., et al. (2009). Protective Effects of Mutant of Acidic Fibroblast Growth Factor against Cerebral Ischaemia-Reperfusion Injury in Rats. Injury 40, 963-967. doi:10. 1016/j.injury.2009.01.110

Xu, X. G., Gong, L., Jiang, T. L., Li, Y. H., Gao, X. H., Tian, H., et al. (2018). Stimulation of Mouse Vibrissal Follicle Growth by Recombinant Human Fibroblast Growth Factor 20. Biotechnol. Lett. 40, 1009-1014. doi:10.1007/ s10529-018-2554-z

Xu, Y. H., Yu, M., Wei, H., Yao, S., Chen, S. Y., Zhu, X. L., et al. (2017). Fibroblast Growth Factor 22 Is a Novel Modulator of Depression through Interleukin1beta. CNS Neurosci. Ther. 23, 907-916. doi:10.1111/cns.12760

Yamasaki, M., Miyake, A., Tagashira, S., and Itoh, N. (1996). Structure and Expression of the Rat mRNA Encoding a Novel Member of the Fibroblast Growth Factor Family. J. Biol. Chem. 271, 15918-15921. doi:10.1074/jbc.271.27.15918

Yang, X., Hui, Q., Yu, B., Huang, Z., Zhou, P., Wang, P., et al. (2018). Design and Evaluation of Lyophilized Fibroblast Growth Factor 21 and its Protection against Ischemia Cerebral Injury. Bioconjug. Chem. 29, 287-295. doi:10. 1021/acs.bioconjchem.7b00588

Yang, Y., and Torbey, M. T. (2020). Angiogenesis and Blood-Brain Barrier Permeability in Vascular Remodeling after Stroke. Curr. Neuropharmacol. 18 (12), 1250-1265. doi:10.2174/1570159X18666200720173316

Yao, D. L., Masonic, K., Petullo, D., Li, L. Y., Lincoln, C., Wibberley, L., et al. (1999). Pretreatment with Intravenous FGF-13 Reduces Infarct Volume and Ameliorates Neurological Deficits Following Focal Cerebral Ischemia in Rats. Brain Res. 818, 140-146. doi:10.1016/s0006-8993(98)01118-4

Yao, X. Y., Li, S., Zhang, L. G., Liu, Z. H., Bao, J. N., and Wu, Z. Y. (2018). Higher Serum Fibroblast Growth Factor-23 Levels and the Risk of Stroke and its Subtypes: Evidence from a Meta-Analysis of Prospective Studies. J. Stroke Cerebrovasc. Dis. 27 (11), 3076-3083. doi:10.1016/j.jstrokecerebrovasdis.2018.06.040
Ye, L., Yang, Y., Zhang, X., Cai, P., Li, R., Chen, D., et al. (2015). The Role of bFGF in the Excessive Activation of Astrocytes Is Related to the Inhibition of TLR4/ NFkB Signals. Int. J. Mol. Sci. 17 (1), 37. doi:10.3390/ijms17010037

Yue, X., Sun, Y., Zhong, M., Ma, Y., Wei, Y., Sun, F., et al. (2018). Decreased Expression of Fibroblast Growth Factor 13 in Early-Onset Preeclampsia Is Associated with the Increased Trophoblast Permeability. Placenta 62, 43-49. doi:10.1016/j.placenta.2017.12.009

Zhang, F., Dai, L., Lin, W., Wang, W., Liu, X., Zhang, J., et al. (2016). Exome Sequencing Identified FGF12 as a Novel Candidate Gene for Kashin-Beck Disease. Funct. Integr. Genomics 16, 13-17. doi:10.1007/s10142-015$0462-\mathrm{Z}$

Zhao, Y. Z., Lin, M., Lin, Q., Yang, W., Yu, X. C., Tian, F. R., et al. (2016). Intranasal Delivery of bFGF with Nanoliposomes Enhances In Vivo Neuroprotection and Neural Injury Recovery in a Rodent Stroke Model. J. Control. Release. 224, 165-175. doi:10.1016/j.jconrel.2016.01.017

Zheng, K., Lin, L., Cui, P., Liu, T., Chen, L., Yang, C., et al. (2020). Association of Fibroblast Growth Factor 23 with Ischemic Stroke and its Subtypes: A Mendelian Randomization Study. Front. Genet. 11, 608517. doi:10.3389/ fgene.2020.608517

Zheng, W., Matei, N., Pang, J., Luo, X., Song, Z., Tang, J., et al. (2019). Delayed Recanalization at 3 Days after Permanent MCAO Attenuates Neuronal Apoptosis through FGF21/FGFR1/PI3K/Caspase-3 Pathway in Rats. Exp. Neurol. 320, 113007. doi:10.1016/j.expneurol.2019.113007

Zheng, X., Zhu, Z., Guo, D., Zhong, C., Xu, T., Peng, Y., et al. (2021). Prognostic Value of Plasma Fibroblast Growth Factor 21 Among Patients with Acute Ischemic Stroke. Eur. J. Neurol. 28 (3), 844-851. doi:10.1111/ene.14683

Zou, R., Zhang, D., Lv, L., Shi, W., Song, Z., Yi, B., et al. (2019). Bioinformatic Gene Analysis for Potential Biomarkers and Therapeutic Targets of Atrial Fibrillation-Related Stroke. J. Transl. Med. 17 (1), 45. doi:10.1186/s12967019-1790-x

Zou, Y., Hu, J., Huang, W., Ye, S., Han, F., Du, J., et al. (2020). Non-Mitogenic Fibroblast Growth Factor 1 Enhanced Angiogenesis Following Ischemic Stroke by Regulating the Sphingosine-1-Phosphate 1 Pathway. Front. Pharmacol. 11, 59. doi:10.3389/fphar.2020.00059

Zuo, S., Zheng, T., and Li, H. (2020). Association between Interleukin-10 -1082A/G Polymorphism and Risk of Ischemic Stroke: A Meta-Analysis. Medicine 99, e18858. doi:10.1097/MD.0000000000018858

Conflict of Interest: The authors declare that the research was conducted in the absence of any commercial or financial relationships that could be construed as a potential conflict of interest.

Copyright (c) 2021 Dordoe, Chen, Huang, Chen, Hu, Wang and Lin. This is an openaccess article distributed under the terms of the Creative Commons Attribution License (CC BY). The use, distribution or reproduction in other forums is permitted, provided the original author(s) and the copyright owner(s) are credited and that the original publication in this journal is cited, in accordance with accepted academic practice. No use, distribution or reproduction is permitted which does not comply with these terms. 